\title{
Calidad de Vida Relacionada con la Salud en Edad Preescolar
}

\author{
ALFONSO URZÚA M. ${ }^{1}$, FRANCISCA MÉNDEZ P. ${ }^{2}$, \\ CAROLINA ACUÑA F. ${ }^{2}$, JOCELYN ASTUDILLO C. ${ }^{2}$ \\ 1. Psicólogo, Magíster en Salud Pública, Doctor en Psicología Clínica y de la Salud. \\ 2. Psicóloga, Licenciada en Psicología. \\ Área Psicología de la Salud, Escuela de Psicología, Universidad Católica del Norte.
}

\begin{abstract}
Quality of Life and Health in Preschoolers

Objective: Parents' perception of quality of life (QOL) was correlated to the health status of children between three and five years eleven months of age. Method: TAPQOL questionnaires were utilized to evaluate parents or guardians of 483 children who attenden prívate, semiprivate and public kindergardens. Average age of the children was 51.7 months, $47 \%$ were girls. Results: QOL was found to be disturbed through anxiety and behavior problems. Some differences were found along the dimensions of gender, type of institution, and presence of illness. Conclusions: Our results allow us to focus on those preschoolers whose parents report a worse QOL, to develop intervention plans. Those are children who have suffered a disease, the youngest, or those who attend public preschools. Positive behavioral signs, anxiety and communication are special areas of interest for medical intervention.
\end{abstract}

(Key words: Quality of life, preschoolers, TAPQOL).

Rev Chil Pediatr 2010; 81 (2): 129-138

\section{RESUMEN}

Objetivo: Bajo un diseño transversal, se evalúa la percepción de los padres sobre la calidad de vida relacionada con la salud (CVRS) en niños entre tres y cinco años once meses. Método: Se aplicó el cuestionario específico de CVRS para menores en edad preescolar TAPQOL a los padres o tutores de 483 infantes que asistían a jardines infantiles particulares, públicos y subvencionados. El promedio de edad de los menores fue de 51,7 meses, siendo el $47 \%$ de estos mujeres. Resultados: La CVRS de los menores se ve más afectada en las dimensiones de ansiedad y problemas de conducta. Se encontraron diferencias en los reportes de las distintas dimensiones dadas por el sexo, el tipo de establecimiento al que acudían los menores y por la presencia de enfermedad. Conclusiones: Los resultados obtenidos nos permiten focalizar la atención en aquellos preescolares

Trabajo recibido el 08 de enero de 2010, devuelto para corregir el 05 de marzo de 2010, segunda versión el 19 de marzo de 2010, aceptado para publicación el 30 de marzo de 2010.

Correspondencia a:

Dr. Alfonso Urzúa M.

E-mail: alurzua@ucn.cl 
cuyos padres reportan una menor CVRS, siendo estos los que han padecido o padecen una enfermedad, los más pequeños y los que asisten a establecimientos públicos, especialmente en las áreas de emociones positivas, ansiedad y comunicación, entregando información para posibles planes de intervención con el objeto de mejorar el bienestar de los menores.

(Palabras clave: Calidad de vida, preescolares, TAPQOL).

Rev Chil Pediatr 2010; 81 (2): 129-138

\section{Introducción}

La calidad de vida (CV) es un concepto que en los últimos años ha cobrado gran importancia. Aun cuando su definición no ha alcanzado un acuerdo generalizado, si se han definido ciertos consensos, tales como que la $\mathrm{CV}$ es subjetiva, que incluye tanto funcionamientos positivos como negativos y que es multidimensional, por lo que debe incorporar diversas facetas o dimensiones de la vida de las personas ${ }^{1,2}$. En este contexto y desde una perspectiva evolutiva, en la población infantil la evaluación de la CV tendría que tener en cuenta la habilidad de participar plenamente en funciones y actividades físicas, sociales y psicosociales apropiadas para cada edad ${ }^{3}$.

La CV en población pediátrica sólo ha comenzado a estudiarse recientemente. Frecuentemente las investigaciones en CV se han centrado en el desarrollo y aplicación del concepto e instrumentos de medida en adultos. En la infancia, la mayoría de las investigaciones se centran en el contexto de enfermedades graves o crónicas ${ }^{4} \mathrm{o}$ en evaluar algunas dimensiones o indicadores relacionados con el concepto tales como el impacto de una enfermedad, la competencia social, el estrés, o las competencias cognitivas ${ }^{5,6}$.

Actualmente, en Iberoamérica son pocas las investigaciones centradas en la $\mathrm{CV}$ en la niñez ${ }^{7,8}$. En población no enferma, en Chile sólo se han encontrado referencias de estudios en población escolar y en la adolescencia ${ }^{9,10}$. No se han reportado estudios que se centren en la $\mathrm{CV}$ o en la calidad de vida relacionada con la salud (CVRS) de los niños en edad preescolar, entendiendo en este grupo a los niños y niñas menores de seis años y que aun no inician su período de educación primaria.
La evaluación temprana de la CVRS en los preescolares permitirá no tan sólo una intervención anticipada en poblaciones y subgrupos demográficos en riesgo, sino que permitirá avanzar en la comprensión del impacto que tiene la salud en la CV de los infantes, pudiéndose de esta manera conocer, planificar, actuar y mejorar la prevención y cuidado a nivel general. Al identificar niveles de morbilidad, permite valorar el impacto sobre la salud de los tratamientos y facilitar la toma de decisiones clínicas, además de profundizar en un concepto relativamente reciente como es, la CVRS en niños/as y adolescentes?

En este marco y considerando la necesidad de incorporar indicadores comprensivos del desarrollo y del bienestar humano en las primeras etapas del desarrollo, esta investigación tuvo por objetivo analizar la CVRS de niños entre tres y cinco años once meses a través de la percepción-evaluación de sus padres o tutores, de diversos dominios de su vida. Pese al relativo consenso en destacar la importancia de no trasladar la visión del adulto a la del niño, pues éste tiene su propia percepción de los eventos vitales que no necesariamente posee el adulto ${ }^{11}$, en el caso de los niños más pequeños, existe un nivel de dependencia que hace necesario que sean las personas responsables de la familia quienes decidan cuándo buscar ayuda en el sistema sanitario. Por este motivo tiene sentido recoger la percepción de calidad de vida del preescolar que tienen las personas responsables del menor ${ }^{12}$.

Se espera encontrar diferencias entre la CVRS dadas por el sexo, en donde los padres reporten que los hombres presentan mayores puntajes en dominios vinculados al desarrollo físico y motor, en tanto las mujeres mayores puntajes en áreas vinculadas a aspectos afec- 
tivos y sociales. También se espera encontrar diferencias en las percepciones de la CVRS según el tipo de establecimiento educacional al que asisten los menores, asignando los padres de los y las menores de establecimientos públicos un menor puntaje en los dominios evaluados con relación a los padres de los establecimientos privados.

\section{Pacientes y Método}

\section{Participantes}

Se realizó un muestreo de tipo intencionado, intentando obtener números similares de participantes en la distribución por sexo y tipo de establecimiento educacional, considerando infantes de jardines infantiles públicos, particulares subvencionados y privados de la ciudad de Antofagasta, todo esto a fin de evaluar las hipótesis de investigación.

Fueron evaluados a través de sus padres o tutores 255 hombres $(52,8 \%)$ y 228 mujeres
$(47,2 \%)$. El promedio de edad de los y las menores fue de 51,7 meses ( $\mathrm{DE}=10,8$ meses), con una edad mínima de 28 meses y una máxima de 71 meses.

\section{Instrumento}

Para evaluar la CVRS se utilizó el cuestionario para menores entre los 3 meses y los 6 años (5 años y 11 meses) de edad llamado TAPQOL. Este cuestionario fue diseñado en Holanda bajo el nombre de TNO-AZL Preschool Children's Quality of Life Questionnaire el año 2001, reportando buenas propiedades psicométricas en su versión original con alfas de cronbach entre, 43 y ,84 en población general $^{13,14}$. Este instrumento fue adaptado al castellano en España ${ }^{12}$, sin embargo, las propiedades psicométricas de esta versión, aún no han sido publicadas. Para la presente investigación, se encontró un alfa de cronbach de ,83 para la escala total.

El TAPQOL es un instrumento multidimensional (tabla 1), orientado a los padres o figuras

\section{Tabla 1. Variables medidas por el TAPQOL}

\begin{tabular}{|c|c|}
\hline Dimensión & Definición \\
\hline \multicolumn{2}{|l|}{ Funcionamiento físico } \\
\hline Sueño (4 ítems) & $\begin{array}{l}\text { Mide las veces que el niño despierta durante la noche y en que condiciones se encuentra } \\
\text { cuando lo hace }\end{array}$ \\
\hline Apetito (3 ítems) & Evalúa si el/la menor ha tenido ganas para comer o no durante los últimos tres meses \\
\hline Respiratorio (3 ítems) & $\begin{array}{l}\text { A partir de esta variable se busca medir si este proceso se ha visto afectado en los últimos } \\
\text { tres meses causando algún malestar al menor }\end{array}$ \\
\hline Digestivo (3 ítems) & $\begin{array}{l}\text { Inquiere sobre la presencia de malestares estomacales que haya tenido el/la menor en los } \\
\text { últimos tres meses }\end{array}$ \\
\hline Piel (3 ítems) & Evalúa las condiciones en que se encuentra esta capa protectora del cuerpo del/ la menor \\
\hline Funcionamiento motor (4 ítems) & Esta variable intenta medir si el niño tiene alguna dificultad para moverse \\
\hline \multicolumn{2}{|l|}{ Funcionamiento social } \\
\hline Funcionamiento social (3 ítems) & Busca medir como se relaciona el niño con sus pares \\
\hline Problemas de conducta (7 ítems) & Evalúa la forma que tiene el niño de reaccionar frente a determinadas situaciones \\
\hline \multicolumn{2}{|l|}{ Funcionamiento cognitivo } \\
\hline Comunicación (4 ítems) & $\begin{array}{l}\text { Intenta medir a partir de esta variable si el niño tiene dificultades para transmitir lo que } \\
\text { piensa o siente y para entender lo que los demás le transmiten }\end{array}$ \\
\hline \multicolumn{2}{|l|}{ Funcionamiento emocional } \\
\hline Vitalidad (3 ítems) & Esta variable busca medir cuanta energía tienen y manifiestan los y las menores \\
\hline Emociones positivas (3 ítems) & Evalúa la intensidad de las reacciones y como estas afectan al niño \\
\hline Ansiedad (3 ítems) & Mide si el/ la menor ha presentado inquietud del ánimo en los últimos tres meses \\
\hline
\end{tabular}


protectoras de los menores, compuesto por 43 ítems configurados en 4 dimensiones y 12 subdimensiones, creadas con base a revisión de la literatura, pruebas empíricas y a la asesoría de psicólogos infantiles, pediatras y padres ${ }^{13}$. En la actualidad este cuestionario se encuentra en proceso de adaptación y validación para la población chilena.

La CVRS, entendida ésta como el nivel de bienestar derivado de la evaluación que la persona realiza de diversos dominios de su vida, considerando el impacto que en éstos tiene su estado de salud ${ }^{15}$, se operacionaliza en el TAPQOL, como la salud de los niños, ponderada por la reacción emocional de los propios niños al estado de sus problemas de salud. En primera instancia se formulan preguntas orientadas a evaluar la existencia de alguna molestia o limitación funcional que pueda tener o que ha presentado el niño en los últimos tres meses y en segunda instancia se presenta una pregunta para detectar la (s) reacción (es) del niño a este tipo de problemas o limitaciones. Cada puntaje por dimensión es convertido mediante una sintaxis de corrección a un puntaje entre $0-100$, en donde un mayor puntaje indica una mejor CVRS.

\section{Procedimientos}

Una vez aprobado el proyecto por el Comité de Bioética de la Universidad Católica del Norte y contando con la autorización de la Junta Nacional de Jardines Infantiles -JUNJIpara los establecimientos públicos, se contactó a 20 instituciones de la ciudad de Antofagasta, solicitando además la autorización de las Directoras de los jardines, en el caso de los establecimientos particulares y subvencionados.

Antes de participar del estudio, se les explicó a los padres de los niños y niñas y a los directivos de los establecimientos intervenidos cual era el objetivo de éste y se les pidió su aprobación con antelación para participar a través de un consentimiento informado.

Fueron repartidos 920 cuestionarios en todos los establecimientos en las reuniones de curso de sus hijos o al momento de retirar a los niños del establecimiento. De éstos fueron devueltos 492, debiéndose eliminar 9 casos por encontrarse fuera del rango de edad de medición propuesto por el instrumento, siendo finalmente válidos para análisis 483 cuestionarios. Una vez recogidos todos los cuestionarios, las respuestas fueron ingresadas a una base de datos construida en SPSS 12.0.

Se describen las medidas de tendencia central de cada una de las dimensiones que componen la escala, tanto a nivel de la muestra total como separadas por sexo y tipo de establecimiento educacional. Para evaluar si existen diferencias entre los distintos subgrupos, se utiliza la prueba $t$ de student para grupos independientes en el caso del sexo, y ANOVA para el caso del tipo de establecimiento. Se analizan también diferencias dadas por el estado de salud reportado por los padres igualmente a través de la prueba t para grupos independientes.

\section{Resultados}

\section{Participantes}

Del total de menores evaluados a través de sus padres o tutores, 176 asistían establecimientos públicos $(36,4 \%), 71$ a establecimientos particulares subvencionados $(14,7 \%)$ y 236 a establecimientos privados $(48,9 \%)$. La distribución de los participantes por sexo y tipo de establecimiento puede ser observada en la tabla 2.

Del total de padres-madres consultados, $256(53 \%)$ reportan que sus hijos estuvieron enfermos en los últimos 3 meses. De la misma manera, 171 fueron reportados como hijos únicos, siendo dos el promedio de número de hermanos de los niños y niñas.

\section{Calidad de vida}

La tabla 3 contiene las medias encontradas en los participantes de este estudio en cada una de las dimensiones evaluadas. Se puede observar que las dimensiones de ansiedad y problemas de conducta son las que los padres reportan como más deficitarias en sus hijos con relación a las otras dimensiones.

\section{CVy variables sociodemográficas}

La tabla 3 asimismo muestra las medias obtenidas en cada dimensión por cada uno de 
Tabla 2. Características de los participantes por tipo de establecimiento

\begin{tabular}{|lcccc|}
\hline & Públicos & Subvencionados & Privados & Total \\
\hline No Hombres (\%) & $91(35,68)$ & $39(15,29)$ & $125(49,01)$ & $255(52,8)$ \\
\hline$N^{0}$ Mujeres (\%) & $85(37,28)$ & $32(14,03)$ & $111(48,68)$ & $228(47,2)$ \\
\hline Edad en meses muestra total $(x \pm D E)$ & $46,51 \pm 8,10$ & $55,42 \pm 10,98$ & $54,41 \pm 11,08$ & $51,68 \pm 10,80$ \\
\hline Edad en meses hombres (x $\pm \mathrm{DE})$ & $45,3 \pm 8,55$ & $54,9 \pm 11,57$ & $53,8 \pm 10,91$ & $51,00 \pm 11,04$ \\
\hline Edad en meses mujeres ( \pm DE) & $47,7 \pm 7,44$ & $55,9 \pm 10,38$ & $55,0 \pm 11,27$ & $52.43 \pm 10,48$ \\
\hline Hijos únicos (\%) & $81(46,02)$ & $28(39,43)$ & $62(26,27)$ & $171(35,40)$ \\
\hline Hospitalizados últimos tres meses $(\%)$ & $3(1,70)$ & $3(4,22)$ & $13(5,50)$ & $19(3,93)$ \\
\hline
\end{tabular}

Tabla 3. Medias y nivel de significación en prueba T para diferencia de medias de las dimensiones del TAPQOL por sexo

\begin{tabular}{|llclc|}
\hline \multicolumn{1}{|c}{ Dimensiones } & Muestra Total & Hombres & Mujeres \\
\hline Digestión & $\mathbf{x} \pm \mathbf{D E}$ & $\mathbf{x} \pm \mathbf{D E}$ & $\mathbf{x} \pm \mathbf{D E}$ & $\mathbf{p}$ \\
\hline Piel & $93,12 \pm 8,2$ & $92,89 \pm 8,1$ & $93,39 \pm 8,4$ & 0,513 \\
\hline Sueño & $95,2 \pm 8,8$ & $94,93 \pm 9,6$ & $95,52 \pm 7,9$ & 0,483 \\
\hline Respiratorio & $89.6 \pm 10,9$ & $88,90 \pm 10,7$ & $90,40 \pm 11,2$ & 0,133 \\
\hline Vitalidad & $92,0 \pm 9,6$ & $91,47 \pm 9,2$ & $92,78 \pm 10,0$ & 0,136 \\
\hline Emoc. positivas & $79,9 \pm 20,7$ & $81,17 \pm 18,6$ & $78,65 \pm 22,9$ & 0,192 \\
\hline Probl. de conducta & $89,5 \pm 22,7$ & $90,52 \pm 21,3$ & $88,55 \pm 24,3$ & 0,345 \\
\hline Ansiedad & $64,2 \pm 23,0$ & $61,94 \pm 24,26$ & $66,86 \pm 21,5$ & 0,019 \\
\hline Func. social & $54,8 \pm 19,1$ & $53,71 \pm 19,7$ & $56,00 \pm 18,5$ & 0,199 \\
\hline Func. motor & $86,5 \pm 21,5$ & $87,32 \pm 20,7$ & $85,78 \pm 22,4$ & 0,436 \\
\hline Comunicación & $97,9 \pm 5,35$ & $97,86 \pm 5,5$ & $98,05 \pm 5,2$ & 0,691 \\
\hline Apetito & $91,3 \pm 10,4$ & $89,85 \pm 11,3$ & $93,12 \pm 9,2$ & 0,001 \\
\hline
\end{tabular}

los sexos. Los hombres presentan una media significativamente menor que las mujeres sólo en las variables problemas de conducta $(\mathrm{t}=$ $-, 2350(480) ; \mathrm{p}<, 05)$ y en comunicación $(\mathrm{t}=$ $-3,494(475,090) ; \mathrm{p}<, 05 ; \mathrm{r}=, 16)$. La medida del tamaño del efecto es baja.

Al analizar la relación existente entre los puntajes obtenidos en las distintas dimensiones y la edad de los participantes, se encuentra que esta correlaciona positivamente con las variables respiratorio $\left(\mathrm{r}=, 16, \mathrm{p}<, 01 ; \mathrm{r}^{2}=\right.$ $, 03)$, sueño $\left(\mathrm{r}=, 0, \mathrm{p}<, 01 ; \mathrm{r}^{2}=, 04\right)$, funcionamiento social $\left(\mathrm{r}=, 10, \mathrm{p}<, 05 ; \mathrm{r}^{2}=, 01\right)$ y comunicación $\left(r=, 25, \mathrm{p}<, 01 ; \mathrm{r}^{2}=, 06\right)$. En todas estas dimensiones a medida que se es mayor, se tiene un mejor puntaje en la dimensión de la calidad de vida evaluada. Asimismo, en todas estas relaciones la medida del tamaño del efecto es baja.

$\mathrm{Al}$ analizar las posibles diferencias en los puntajes dadas por la estructura familiar, no se encuentran diferencias estadísticamente significativas en los puntajes reportados entre aquellos que son hijos únicos y aquellos que tienen hermanos. Pese a esto, se encuentra relación entre el puntaje obtenido y el número de hermanos, en donde este último correlacionó de manera negativa con las dimensiones vitalidad $\left(\mathrm{r}=-, 13, \mathrm{p}<, 05 ; \mathrm{r}^{2}=, 02\right)$ y funcionamiento motor $\left(\mathrm{r}=-, 14, \mathrm{p}<, 05, \mathrm{r}^{2}=, 02\right)$. En ambos ca- 
sos, el puntaje atribuido a esta dimensión disminuye a medida que se acrecienta el número de hermanos, siendo bajo en ambas relaciones el tamaño del efecto.

$\mathrm{Al}$ analizar las diferencias en las medias dadas por el tipo de establecimiento al que acude el o la menor (tabla 4), se encuentran diferencias estadísticamente significativas sólo en las variables emociones positivas ( $\mathrm{f}=4798$ (2 472), $\mathrm{p}<, 05 ; \mathrm{r}=, 95)$; ansiedad ( $\mathrm{f}=3542$ (2 457), $\mathrm{p}<, 05, \mathrm{r}=, 91)$ y comunicación ( $\mathrm{f}=$ 10641 (2 478), $\mathrm{p}<, 01, \mathrm{r}=$,99). En todas las anteriores se observa un alto tamaño del efecto.

Al realizar la prueba posthoc de Bonferroni, se encuentra que en la dimensión de emociones positivas, la diferencia significativa se encuentra entre las medias de los menores de los establecimientos particulares y los que asisten a los establecimientos públicos, en donde, la media de estos últimos es significativamente menor en esta dimensión $(\mathrm{p}<, 01)$.

En la dimensión ansiedad, las diferencias se encuentran entre las medias de los menores que acuden a establecimientos subvencionados y los que asisten a establecimientos públicos, siendo la media de estos últimos significativamente mayor que la media de los menores que acuden a un establecimiento subvencionado ( $p$ $<, 05)$.
En la dimensión comunicación, se encuentran diferencias significativas entre los establecimientos privados y los particulares $(\mathrm{p}<$ ,01) y los subvencionados ( $\mathrm{p}<, 01)$, siendo la media de los menores que acuden a establecimientos públicos significativamente menor que los otros tipos de establecimientos.

\section{Calidad de vida y salud}

La tabla 5 muestra las medias obtenidas en las dimensiones dadas por el estado de salud del o la menor al responder el padre/madre si el menor ha estado enfermo o no en los últimos tres meses.

Al comparar ambos grupos, se puede observar que existen diferencias estadísticamente significativas en las variables funcionamiento digestivo $(\mathrm{t}=-4,66(473), \mathrm{p}<, 01 ; \mathrm{r}=, 21)$, piel $(\mathrm{t}=-, 2532(409,752), \mathrm{p}<, 05, \mathrm{r}=, 01)$, sueño $(\mathrm{t}=-3,246(472,311), \mathrm{p}<, 01, \mathrm{r}=, 15)$, apetito $(\mathrm{t}=-3,748(472), \mathrm{p}<, 01, \mathrm{r}=, 17) ;$ conducta $(\mathrm{t}$ $=-3,889(479,992), \mathrm{p}<, 01, \mathrm{r}=, 18)$ y ansiedad $(\mathrm{t}=-2,145(458), \mathrm{p}<, 05, \mathrm{r}=, 09)$, todas ellas con un bajo tamaño del efecto, y en la variable respiración $(\mathrm{t}=-9,43(410,176) ; \mathrm{p}<, 01, \mathrm{r}=$ ,42), con un tamaño del efecto medio.

En todas estas dimensiones, la media reportada por los padres de aquellos que han estado enfermos los últimos tres meses son significa-

Tabla 4. Medias y nivel de significación del ANOVA para la diferencia entre medias de las dimensiones del TAPQOL por tipo de establecimiento

\begin{tabular}{|lcccc|}
\hline & \multicolumn{1}{c}{ Privado } & Subvencionado & Público \\
\hline Digestión & $\mathbf{x} \pm \mathbf{D E}$ & $\mathbf{x} \pm \mathbf{D E}$ & $\mathbf{x} \pm \mathbf{D E}$ & $\mathbf{P}$ \\
\hline Piel & $93,23 \pm 8,35$ & $91,55 \pm 8,85$ & $93,63 \pm 7,76$ & 0,195 \\
\hline Respiración & $95,34 \pm 9,19$ & $95,54 \pm 8,55$ & $94,88 \pm 8,44$ & 0,829 \\
\hline Sueño & $92,52 \pm 8,67$ & $93,19 \pm 8,83$ & $91,03 \pm 10,10$ & 0,177 \\
\hline Vitalidad & $90,40 \pm 10,52$ & $90,32 \pm 10,60$ & $88,26 \pm 11,58$ & 0,123 \\
\hline Emoc. positivas & $79,37 \pm 20,85$ & $81,22 \pm 16,17$ & $80,28 \pm 22,39$ & 0,783 \\
\hline Probl. de conducta & $92,17 \pm 18,54$ & $91,31 \pm 20,48$ & $85,29 \pm 27,90$ & 0,009 \\
\hline Ansiedad & $66,25 \pm 21,18$ & $65,39 \pm 24,23$ & $61,10 \pm 24,76$ & 0,074 \\
\hline Func. social & $55,17 \pm 18,14$ & $49,53 \pm 18,47$ & $56,69 \pm 20,49$ & 0,030 \\
\hline Func. motor & $87,39 \pm 20,98$ & $90,14 \pm 16,80$ & $84,02 \pm 23,66$ & 0,095 \\
\hline Comunicación & $98,14 \pm 5,50$ & $98,33 \pm 5,06$ & $97,55 \pm 5,28$ & 0,443 \\
\hline Apetito & $92,96 \pm 9,84$ & $93,22 \pm 8,82$ & $88,52 \pm 11,38$ & 0,000 \\
\hline
\end{tabular}


tivamente menores que las que no lo han estado.

Al comparar las medias de los que han reportado haber estado hospitalizados en algún período los últimos tres meses con los que no (tabla 5), sólo se encuentra diferencias estadísticamente significativas en la variable de funcionamiento respiratorio, en donde la media de los que han estado hospitalizados es significativamente menor en esta dimensión que la de aquellos que no lo han estado.
$\mathrm{Al}$ realizar un análisis de varianza factorial a través del procedimiento modelo lineal general univariante, considerando como variable dependiente cada una de las dimensiones, y como factores fijos el sexo, tipo de establecimiento, la presencia de enfermedad y la hospitalización, se encuentra que el efecto de estos factores sólo es significativa a algunas dimensiones. La tabla 6 muestra sólo las dimensiones en las cuales los efectos del modelo (efecto de los factores, el de la interacción y el de la

Tabla 5. Medias y nivel de significación para pruebas $\mathbf{T}$ de diferencias de medias por presencia de enfermedad y hospitalización

\begin{tabular}{|c|c|c|c|c|c|c|}
\hline & $\begin{array}{l}\text { Enfermo } \\
x \pm D E\end{array}$ & $\begin{array}{c}\text { No enfermo } \\
x \pm D E\end{array}$ & $\mathbf{p}$ & $\begin{array}{l}\text { Hospitalizado } \\
\qquad \pm \pm D E\end{array}$ & $\begin{array}{c}\text { No hospitalizado } \\
x \pm D E\end{array}$ & $\mathbf{p}$ \\
\hline Digestivo & $91,5 \pm 8,75$ & $94,9 \pm 7,19$ & 0,000 & $89,47 \pm 11,73$ & $93,27 \pm 8,03$ & 0,178 \\
\hline Piel & $94,2 \pm 10,27$ & $96,2 \pm 6,68$ & 0,012 & $90,74 \pm 12,08$ & $95,3 \pm 8,61$ & 0,124 \\
\hline Respiración & $88,54 \pm 10,68$ & $96,0 \pm 6,24$ & 0,000 & $84,72 \pm 14,36$ & $92,37 \pm 9,27$ & 0,038 \\
\hline Sueño & $88,11 \pm 12,05$ & $91,2 \pm 9,30$ & 0,001 & $86,84 \pm 9,96$ & $89,71 \pm 10,98$ & 0,262 \\
\hline Vitalidad & $81,3 \pm 19,40$ & $78,38 \pm 22,16$ & 0,123 & $80,19 \pm 20,42$ & $84,25 \pm 33,07$ & 0,247 \\
\hline Emoc. positivas & $90,46 \pm 21,15$ & $88,59 \pm 24,46$ & 0,371 & $89,78 \pm 22,30$ & $89,78 \pm 22,30$ & 0,313 \\
\hline Probl. de conducta & $60,48 \pm 23,48$ & $68,49 \pm 21,26$ & 0,000 & $54,58 \pm 28,12$ & $64,65 \pm 22,78$ & 0,062 \\
\hline Ansiedad & $53,01 \pm 18,18$ & $56,83 \pm 19,98$ & 0,032 & $49,07 \pm 22,48$ & $55,05 \pm 18,97$ & 0,194 \\
\hline Func. social & $86,56 \pm 20,50$ & $86,62 \pm 22,63$ & 0,976 & $82,45 \pm 21,85$ & $86,76 \pm 21,49$ & 0,393 \\
\hline Func. motor & $97,77 \pm 5,80$ & $98,14 \pm 4,80$ & 0,471 & $98,02 \pm 4,68$ & $97,94 \pm 5,38$ & 0,950 \\
\hline Comunicación & $90,89 \pm 10,67$ & $91,94 \pm 10,27$ & 0,276 & $94,07 \pm 8,70$ & $91,27 \pm 10,55$ & 0,255 \\
\hline Apetito & $85,34 \pm 12,86$ & $89,63 \pm 11,90$ & 0,001 & $82,87 \pm 11,94$ & $87,51 \pm 12,59$ & 0,125 \\
\hline
\end{tabular}

Tabla 6. Análisis de varianza factorial para las dimensiones de calidad de vida

\begin{tabular}{|c|c|c|c|c|}
\hline & $\mathbf{R}$ & $\mathbf{R}^{2}$ corregida & $\mathbf{F}$ & $\mathbf{p}$ \\
\hline Digestión & 0,087 & 0,051 & & \\
\hline Enfermedad & & & 6,172 & 0,013 \\
\hline Respiración & 0,217 & 0,186 & & \\
\hline Enfermedad & & & 7,739 & 0,006 \\
\hline Sexo + tipo de establecimiento & & & 3,027 & 0,049 \\
\hline Enfermedad + tipo de establecimiento & & & 3,716 & 0,025 \\
\hline Sexo + tipo de establecimiento + hospitalización & & & 4,333 & 0,038 \\
\hline Conducta & 0,107 & 0,072 & & \\
\hline Tipo de establecimiento & & & 3,372 & 0,035 \\
\hline Enfermedad + tipo de establecimiento & & & 5,447 & 0,005 \\
\hline Comunicación & 0,100 & 0,065 & & \\
\hline Sexo + tipo de establecimiento & & & 3,339 & 0,036 \\
\hline
\end{tabular}


constante o intersección) fueron significativos y adicionalmente en cada dominio, los factores cuyos efectos al interior de cada modelo fueron significativos.

Tal como se puede apreciar, el dominio cuya varianza es mayormente explicado por los factores incluidos en el modelo es la respiración, en donde el efecto de los cuatro factores incorporados, son capaces de explicar un $18,6 \%$ de la varianza de esta variable.

\section{Discusión}

Los instrumentos para la evaluación de la CVRS en población infantil y adolescente han cobrado una gran relevancia en la última década, ya que permiten captar la multidimensionalidad de la salud ${ }^{16}$. En este estudio, los datos obtenidos a partir del TAPQOL entregan información valiosa sobre diversas dimensiones relacionadas con la $\mathrm{CV}$ de los menores evaluados a través de sus padres. Al comparar los resultados de este estudio con los realizados en España y Holanda se puede afirmar que existen similitudes en relación a la mayoría de las dimensiones medidas. Sin embargo, existen diferencias en relación a vitalidad, ansiedad, problemas de conducta y funcionamiento social, donde la CV reportada para los preescolares chilenos es menor. Esto podría deberse a las características propias de las culturas de cada uno de estos tres grupos, puesto que se ha señalado que muchos de los problemas que persisten o se agravan en el ámbito de la salud infantil están relacionados con el estilo de vida, es decir, con patrones o formas de vivir que se conforman por el contexto social y físico en el que están insertos los niños, pudiendo esto ser una posible fuente de las diferencias encontradas ${ }^{17}$.

Se esperaba encontrar diferencias en la CVRS reportada para niños y niñas. Las mayores diferencias se encontraron en el ámbito de la comunicación y la conducta, siendo estas las áreas que los padres reportan más afectan la $\mathrm{CV}$ de las niñas. Esto podría deberse a que las mujeres en esta etapa de su vida, priorizan más que los niños el ámbito de la socialización, ámbito en el que la comunicación y la con- ducta adquieren una gran relevancia, al influir directamente en las relaciones entre pares ${ }^{10}, o$ bien, derivados de brechas con las expectativas de los padres acerca de lo que debe ser el normal funcionamiento o conductas para un determinado sexo.

Se encontró que a medida que avanza la edad de los niños, la CV reportada por los padres aumenta, al percibir estos una mejora en el funcionamiento social, respiratorio, sueño, y en la comunicación. Estos resultados contrastan con otros estudios quienes reportan que a medida que avanza la edad disminuye la $\mathrm{CV}$ de los niños, en todas las dimensiones evaluadas $^{10}$. Es posible que esta diferencia se deba a que en el presente estudio la información es entregada por los padres mientras que en otros la información es entregada directamente por los niños. Adicionalmente, las dimensiones evaluadas son distintas, teniendo énfasis el TAPQOL en el impacto del estado de salud de los menores, los que a menor edad son más vulnerables físicamente, sumado a que en la evaluación de los menores con mayor edad, se evalúan otros ámbitos que incorporan no tan sólo la salud, sino además la interacción cada vez más activa del menor con su medio externo (padres, amigos, escuela). Por otra parte, al ser los padres quienes reportan respecto de la $\mathrm{CV}$ de sus hijos, la información entregada puede encontrarse sesgada, ya que, se refiere a la información percibida por estos, o bien puede estar mediada por la deseabilidad de ver a sus hijos sanos.

Otro aspecto indagado fue la relación entre la CV y el número de hermanos, en donde se evidenció que a mayor cantidad de hermanos disminuye la vitalidad y el funcionamiento motor de los preescolares. Al respecto, no se han encontrado otros estudios que permitan hacer una comparación sobre el tema. Posiblemente al aumentar el número de hermanos a los padres o figuras protectoras les es más difícil entregar el tiempo y la atención necesaria a cada uno de sus hijos en el ámbito físico, social y emocional en esta etapa, lo que podría influir en el estado de salud de los niños, por lo cual pueden llegar a enfermarse más, lo que contribuiría además a disminuir su vitalidad y funcionamiento motor. Se sugiere frente a estas 
conjeturas profundizar con nuevos estudios a modo de poder diseñar estrategias de intervención adecuadas frente a las dimensiones que aparecen como debilitadas, siendo necesario contrastar estos resultados con otras investigaciones que midan el mismo grupo etáreo, con similar metodología y dimensiones.

$\mathrm{Al}$ indagar en la relación existente entre el tipo de establecimiento educacional y la CV, se encontraron similitudes en los reportes de los padres-madres en la mayoría de las dimensiones medidas en este estudio. Sin embargo, se evidenciaron puntajes menores en las dimensiones sobre emociones positivas, ansiedad y comunicación en establecimientos públicos, lo que implica la percepción parental de una menor CV de los niños que asisten a este tipo de establecimiento. Esto podría deberse a varios factores, pudiendo existir verdaderamente una menor $\mathrm{CV}$ en los menores que asistían a establecimientos públicos, tal como se ha reportado para similar tipo de establecimiento en escolares de enseñanza básica y media ${ }^{9,10}$. Esto se ha vinculado en edades mayores a variables como al nivel educacional de los padres, el tipo de relación existente entre ellos, o bien el tipo y desarrollo de las habilidades parentales, los cuales podrían estar disminuidos en los padres pertenecientes a sectores más deprivados socialmente, repercutiendo directamente en el desarrollo socioemocional de los menores evaluados?.

En relación a los niños que han estado enfermos, se encontró que las dimensiones que los padres consideran que más afectan la $\mathrm{CV}$ son el funcionamiento digestivo, piel, respiración, sueño, apetito, conducta y ansiedad. Probablemente esto se deba a que al estar enfermos, los padres perciben claramente en los menores los síntomas derivados de un malestar físico generalizado, el cual afecta tanto su ciclo de sueño como sus hábitos alimentarios y también su conducta. Por otra parte, el tener que permanecer en cama para guardar reposo o el tener que disminuir su actividad física genera que los niños se tornen más ansiosos e inquietos.

Con respecto al funcionamiento digestivo y la respiración, se puede mencionar que estas son enfermedades frecuentes a nivel nacio- nal en los niños de esta edad ${ }^{18}$, mientras que en relación a la piel, esto puede deberse a las características especiales con que cuenta la región de Antofagasta, donde han sido detectados los índices de radiación y contaminación más altos a lo largo de este último tiempo a nivel nacional. Por otra parte, en relación a los niños que han estado hospitalizados en los últimos tres meses se encontró que la dimensión que más afectó su $\mathrm{CV}$ es el funcionamiento respiratorio. Esto podría explicarse porque el período de recolección de datos fue durante la temporada invernal, época del año en que aumentan las enfermedades respiratorias, además de la presencia de la pandemia de AH1N1 que afectó a gran parte de la población infantil.

En relación a las limitaciones de este estudio, es importante mencionar que la muestra fue recogida durante el período de invierno, siendo este uno de los más críticos para nuestro país, en lo que a salud se refiere, debido a que gran parte de la población chilena se vio afectada por la influenza humana (AH1N1), clasificada como pandemia. Por otra parte, se considera como limitación el desconocimiento del sexo y la edad de quienes contestaron el cuestionario, de manera que para futuros estudios, se sugiere incluir la edad y el sexo del padre o tutor del menor que conteste el cuestionario de manera de poder establecer diferencias entre lo que reportan los padres y lo que reportan las madres.

Respecto a las fortalezas podemos señalar que nos brinda la oportunidad de detectar las causas que están afectando directamente la CV en los niños preescolares, lo que a su vez permitiría realizar intervenciones tempranas, para mejorar la $\mathrm{CV}$ en la etapa adulta, relevando paralelamente la importancia en la primera infancia de los contextos más inmediatos y tempranos, como el familiar, ya que a medida que este crece, otros contextos relacionales van adquiriendo mayor relevancia.

\section{Referencias}

1.- Bullinger M: Quality of life: Definition, conceptualization and implications: a methodologists view. Theor Surg 1991; 6: 143-8. 
URZÚA A. y cols.

2.- Patrick D, Erickson P: Health Policy, Quality of Life: Health Care Evaluation and Resource Allocation. Oxford University Press. 1993. New York.

3.- Starfield B: Measurement of outcome: a proposed scheme. Milbank Mem Fund 1974: Q; 52, 39-50.

4.- Sabeh E, Verdugo M: Evaluación de la percepción de calidad de vida en la infancia. Psicothema 2002; 14 : 86-91.

5.- Rajmil L, Herdman M, Fernández de Sanmamed M, et al, Kidscreen group: Generic health-related quality of life instruments in children and adolescents: a qualitative analysis of content. J Adolesc Health 2004; 34: 37-45.

6.- Solans M, Pane S, Estrada M, et al: Health-related quality of life measurement in children and adolescents: A systematic review of generic and disease-specific instruments. Value Health 2008; 11: 742-64.

7.- Rajmil L, Roizen M, Urzúa A: Calidad de Vida y salud en la infancia y la adolescencia. Revista Típica 2009 (en prensa).

8.- Rajmil L, Estrada MD, Herdman M, Serra-Sutton V, Alonso J: Calidad de vida Relacionada con la salud (CVRS) en la infancia y la adolescencia: Revisión de la bibliografía y de los instrumentos adaptados en España. Gac Sanit 2001; 15: 34-43.

9.- Urzúa A, Cortés E, Prieto L, Vega S: Autoreporte de la calidad de vida en niños y adolescentes escolarizados. Rev Chil Pediatr 2009; 80: 238-44.

10.- Urzúa A, Mercado G: La evaluación de la calidad de vida de los y las adolescentes a través del Kiddo- Kindl. Terapia Psicológica 2008; 26: 133-41.

11.- Casas F: Terapia Psicológica: La evaluación de calidad de vida de niños y niñas. Anuario de Psicología 1992; 53: 27-45.

12.- Sardón O, Morera G, Herdman M, et al: Versión española del TAPQOL: Calidad de vida relacionada con la salud en niños de 3 meses a 5 años. An Pediatr (Barc) 2008; 68: 420-4.

13.- Fekkes M, Theunissen N, Brugman E, et al: Development and psychometric evaluation of the TAPQoL: a health related quality of life instrument for 1-5 old year children. Quality of Life Research 2000; 9: 96172.

14.- Bunge EM, Essink-Bot M, Kobussen M., van SuijlekomSmit L, Moll H, Raat H: Reliabilitry and validity of health status measurement by the TAPQOL. Arch Dis Child 2005; 90: 351-8.

15.- Urzúa A: Calidad de Vida relacionada con la salud: aspectos conceptuales. Rev Med Chile 2010; 138: 341-8.

16.- Aymerich M, Berra S, Guillamon I, Herdman M, Alonso $J$, Ravens-Sieberer U: Desarrollo de la versión en español del KIDSCREEN, un cuestionario de calidad de vida para la población infantil y adolescente. Gac Sanit 2005; 19: 93-102.

17.- Colomer C, Colomer J, Mercer R, Peiró R, Rajmil L: La salud en la infancia. Gac Sanit 2004; 18: 39-45.

18.- Pinto R: Estado actual de asma en el niño. Rev Chil Enfer Respir 2000; 11: 145-48. 